\title{
Tissue Spillover Correction for Dynamic Pinhole SPECT Studies of Fatty Acid Metabolism in the Rat Heart
}

\author{
Bryan W. Reutter, Senior Member, IEEE, Rostyslav Boutchko, Member, IEEE, \\ Ronald H. Huesman, Fellow, IEEE, Anne C. Sauve, and Grant T. Gullberg, Fellow, IEEE
}

\begin{abstract}
The goal of this research is to quantitatively compare fatty acid metabolism in the hearts of Wistar-Kyoto (WKY) normal rats and spontaneously hypertensive rats (SHR) as a function of age, and thereby track physiological changes associated with the onset and progression of heart failure in the SHR model. The fatty acid analog, ${ }^{123}$ I-labeled BMIPP, was used in longitudinal dynamic pinhole SPECT imaging studies performed on two WKY normal rats and two SHRs every seven months for 21 months. In previous work, we addressed issues associated with reconstructing dynamic data acquired with a slowly rotating camera. In this work, we address quantitative effects of limited spatial resolution that result in underestimation of metabolic rate from compartmental models. In particular, blurring of activity between the left ventricular blood pool and surrounding myocardial tissue decreases contrast between blood input and tissue uptake time-activity curves (TACs). Standard compartmental modeling straightforwardly accounts for spillover of blood activity into tissue volumes. However, accounting for spillover of tissue activity into blood volumes is more problematic. Because of tissue spillover, there is no reconstructed voxel that contains a pure blood TAC. Thus, we developed a method to jointly estimate the pure blood input along with compartmental model parameters from B-spline TACs reconstructed directly from dynamic SPECT projection data for 11 studies. Tissue spillover correction improved the contrast between blood input and myocardial uptake curves for all studies and visually improved the fit of the compartmental model for some studies. Estimates of metabolic rate of ${ }^{123}$ I-labeled BMIPP increased by an average of $72 \pm 45 \%$ across all 11 studies, compared to estimates obtained without spillover correction. Thus, the tissue spillover correction method resulted in improved quantitative dynamic imaging of fatty acid metabolism in the rat heart, even with slow camera rotation.
\end{abstract}

\section{INTRODUCTION}

$\mathbf{T}$ HE overarching goal of this research is to quantitatively compare fatty acid metabolism in the hearts of WistarKyoto (WKY) normal rats and spontaneously hypertensive rats (SHR) as a function of age, and thereby track physiological changes associated with the onset and progression of heart failure in the SHR model. The fatty acid analog, ${ }^{123}$ I-labeled

This work was supported by the National Institutes of Health of the U. S. Department of Health and Human Services under grants R01-EB00121, R01EB007219, and R01-HL71253; and by the Director, Office of Science, Office of Biological and Environmental Research of the U. S. Department of Energy under contract DE-AC02-05CH11231.

B. W. Reutter, R. Boutchko, R. H. Huesman, A. C. Sauve, and G. T. Gullberg are with the Department of Radiotracer Development \& Imaging Technology, Lawrence Berkeley National Laboratory, One Cyclotron Road, Berkeley, CA 94720, USA (e-mail: bwreutterelbl.gov).
BMIPP, was used in longitudinal metabolic imaging studies performed every seven months for 21 months.

In previous work, we addressed issues associated with reconstructing dynamic data acquired with use of a slowly rotating camera [1]-[3]. The work presented here addresses quantitative effects of limited spatial resolution in dynamic pinhole SPECT that result in underestimation of the metabolic rate of ${ }^{123}$ I-BMIPP in the rat myocardium. In particular, blurring of activity between the left ventricular blood pool and surrounding myocardial tissue decreases contrast between blood input and tissue uptake time-activity curves (TACs). Standard compartmental modeling straightforwardly accounts for the spillover of blood activity into tissue volumes [4]. However, accounting for the spillover of tissue activity into blood volumes is more problematic, and is the focus of the work presented here. Results are presented for imaging studies performed on two WKY normal rats and two SHRs when the rats were age 7,14 , and 21 months.

\section{Methods}

\section{A. Pinhole SPECT Data Acquisition and System Modeling}

With use of methods described in [5], dynamic cardiac pinhole SPECT projection data and pinhole geometric calibration data were acquired with slow gantry rotation on a dual-head GE Millennium VG Hawkeye SPECT/CT scanner equipped with custom pinhole collimators (Fig. 1). For each study, an injection of about $4 \mathrm{mCi}$ of ${ }^{123} \mathrm{I}$-BMIPP was performed shortly after the dynamic data acquisition began. Data were acquired for $60 \mathrm{~min}$ in 1-sec time frames with an angular step of 4 degrees per frame. Collimator response was modeled via ray tracing and excluded the effects of collimator penetration. The system model also excluded the effects of attenuation and scatter; however, we are currently studying these effects via Monte Carlo simulation in a separate investigation [6], [7].

\section{B. Fully 4-D Dynamic SPECT Image Reconstruction}

The time-varying spatial distribution of ${ }^{123}$ I-BMIPP is modeled with use of 4-D multiresolution B-splines that are piecewise constant in space and piecewise quadratic in time. The 4-D splines are spatially organized on a $10 \times 10 \times 10$ $3-\mathrm{D}$ grid that provides uniform sampling of $6.4 \mathrm{~mm}$ in each dimension. Inside the volume containing the heart, a $3 \times 3 \times 3$ neighborhood of these lower-resolution splines is replaced by a $12 \times 12 \times 12$ neighborhood of higher-resolution splines that 

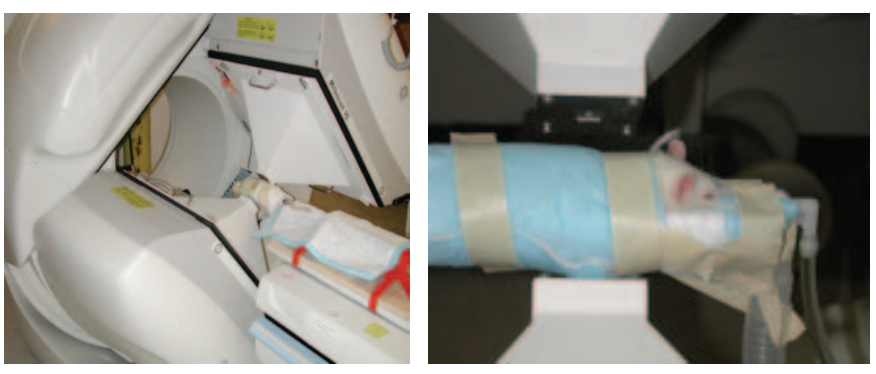

Fig. 1. Clinical dual-head SPECT/CT scanner with custom pinhole collimators used for quantitative dynamic imaging of fatty acid metabolism in the rat heart.

provide uniform sampling of $1.6 \mathrm{~mm}$. The 4-D splines are temporally organized on a $1-\mathrm{D}$ grid that provides nonuniform sampling intervals of 0-2.4, 2.4-9.4, 9.4-30, and 30-90 sec during the first gantry rotation (Fig. 2).

With use of a fully 4-D algorithm for penalized weighted least-squares image reconstruction that we developed, Bspline TACs for the multiresolution voxels are estimated directly from the dynamic pinhole SPECT projection data [3]. This yields estimates of temporal B-spline coefficients $\left\{\hat{a}_{m n} ; m=1, \ldots, M ; n=1, \ldots, N\right\}$, where $M$ is the number of spatial voxels and $N$ is the number of temporal B-spline basis functions. The estimated TAC for the $m$-th voxel is

$$
\hat{A}^{m}(t)=\sum_{n=1}^{N} \hat{a}_{m n} V^{n}(t),
$$

where $V^{n}(t)$ are temporal B-spline basis functions (Fig. 2).

\section{Joint Estimation of Blood Input and Fatty Acid Metabolism}

To obtain a quantitative estimate of the metabolic rate of ${ }^{123} \mathrm{I}$-BMIPP in the myocardium, a one-tissue-compartment model (Fig. 3) is fitted to TACs for higher-resolution voxels in a $7 \times 7 \times 7$ neighborhood centered on the blood pool. Early myocardial tissue uptake is modeled with a single, irreversible compartment:

$$
\operatorname{tissue}(t)=K_{i} \cdot \int_{0}^{t} \operatorname{blood}(\tau) d \tau,
$$

where $K_{i}$ is the metabolic rate of ${ }^{123}$ I-BMIPP. Each voxel is modeled as a mixture of blood input and tissue uptake:

$$
\operatorname{voxel}(t)=\left[f_{v} \cdot \operatorname{blood}(t)\right]+\left[\left(1-f_{v}\right) K_{i} \cdot \int_{0}^{t} \operatorname{blood}(\tau) d \tau\right]_{(3)},
$$

where $f_{v}$ is the fraction of vasculature in the tissue and also incorporates the effect of spillover from the blood pool to surrounding tissue voxels. For blood voxels, the factor $\left(1-f_{v}\right) K_{i}$ incorporates the effect of spillover from surrounding tissue voxels.

Because of tissue spillover, there is no reconstructed voxel that contains a pure blood TAC; thus, the blood input function is assumed to be unknown and is modeled by a B-spline TAC:

$$
B(t)=\sum_{n=1}^{N} b_{n} V^{n}(t)
$$

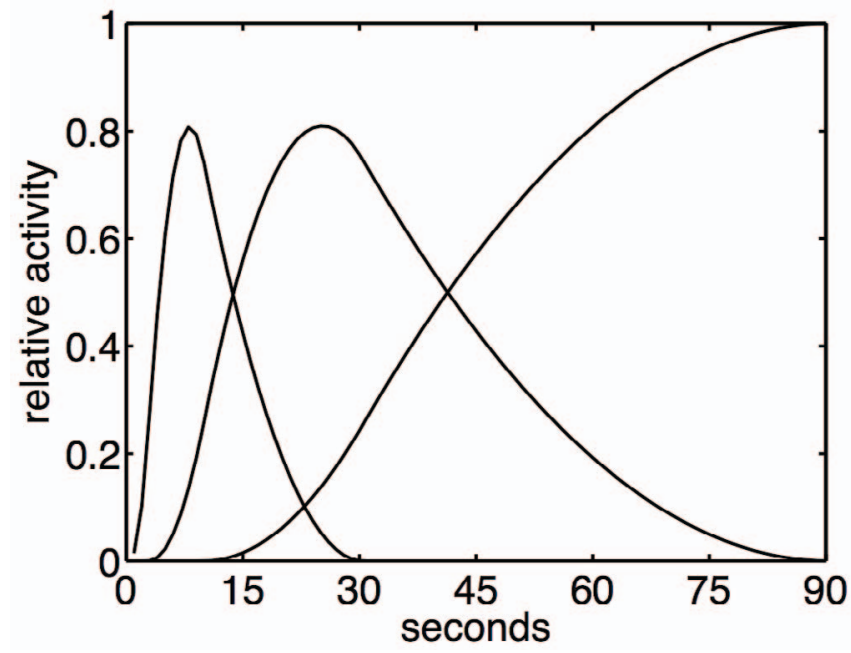

Fig. 2. Piecewise quadratic temporal B-spline basis functions used to reconstruct dynamic data acquired during the first gantry rotation.

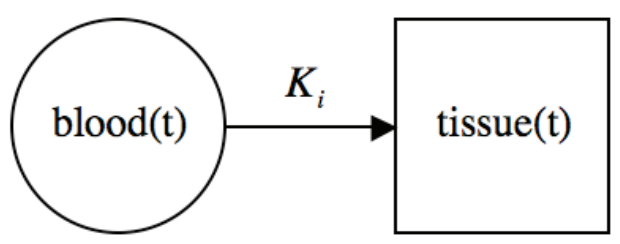

Fig. 3. One-tissue-compartment model used for quantifying fatty acid metabolism during the first $90 \mathrm{sec}$ after injection of ${ }^{123}$ I-BMIPP.

The temporal B-spline coefficients $\left\{b_{n} ; n=1, \ldots, N\right\}$ are jointly estimated with compartmental model parameters $\left\{\left(f_{v}^{m}, k^{m}\right) ; m \in \Omega\right\}$ for each voxel by minimizing the following least-squares criterion:

$$
\sum_{m \in \Omega} \int_{0}^{T}\left\{\hat{A}^{m}(t)-\left[f_{v}^{m} B(t)+k^{m} \int_{0}^{t} B(\tau) d \tau\right]\right\}^{2} d t
$$

where $\Omega$ denotes the $7 \times 7 \times 7$ neighborhood of voxels centered on the blood pool, $T=90 \mathrm{sec}$, and $k^{m}=\left(1-f_{v}^{m}\right) K_{i}^{m}$. Thus, there is a total of $\left(2 \cdot 7^{3}\right)+3=689$ parameters to jointly estimate.

The minimization proceeds by first initializing $B(t)$ to the TAC for the voxel at the center of the neighborhood $\Omega$. Then, optimal values for the B-spline coefficients $\left\{b_{n} ; n=1, \ldots, N\right\}$ for $B(t)$ are found with use of an iterative search algorithm. Note that one does not need to search explicitly for optimal values for the conditionally linear compartmental model parameters $\left\{\left(f_{v}^{m}, k^{m}\right) ; m \in \Omega\right\}$, as there are unique optimal values for these parameters given $B(t)$ [8], [9].

Values for $K_{i}$ reported in the results are based on the final estimate for $B(t)$ and the average of TACs for 12 myocardial tissue voxels identified in static 3-D images reconstructed from summed late data acquired 1.5-60 min after injection (Fig. 4).

\section{RESULTS}

The spillover of tissue activity into the left ventricular blood pool averaged $19 \pm 10 \%$ across all 11 studies. Tissue spillover 


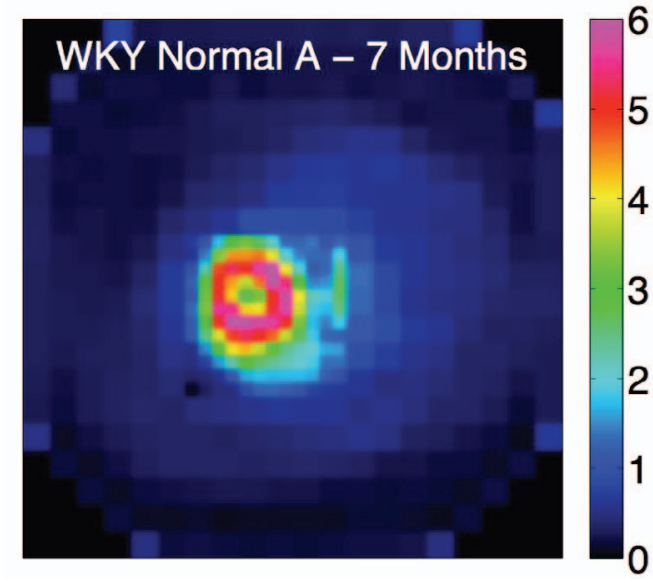

Fig. 4. Trapping of ${ }^{123}$ I-BMIPP in the myocardium is evident in this midventricular slice through a late 3-D static image of a WKY normal heart.

SHR A, 7 Months: $\mathrm{K}_{\mathrm{i}}=0.64 / \mathrm{min}$

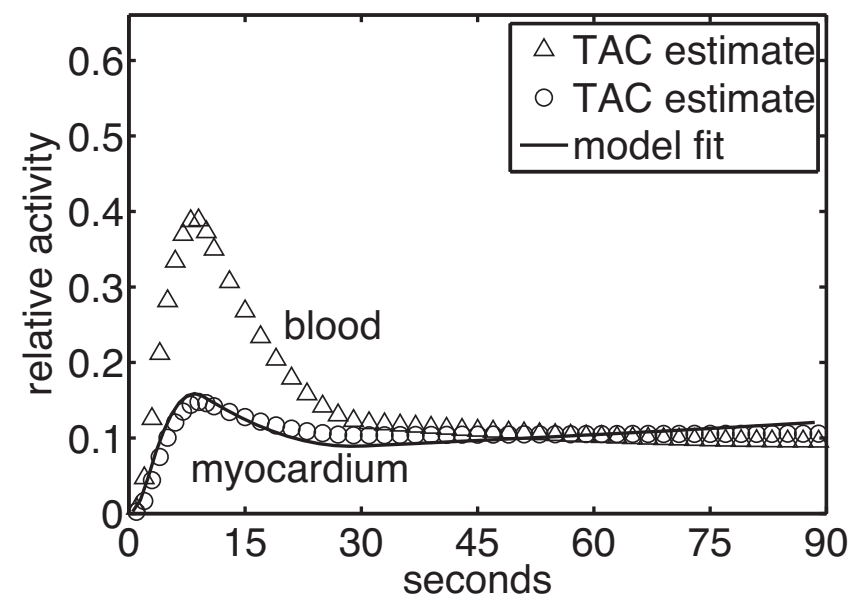

(a) blood curve not corrected for spillover from myocardium

SHR A, 7 Months: $K_{i}=1.12 / \mathrm{min}$

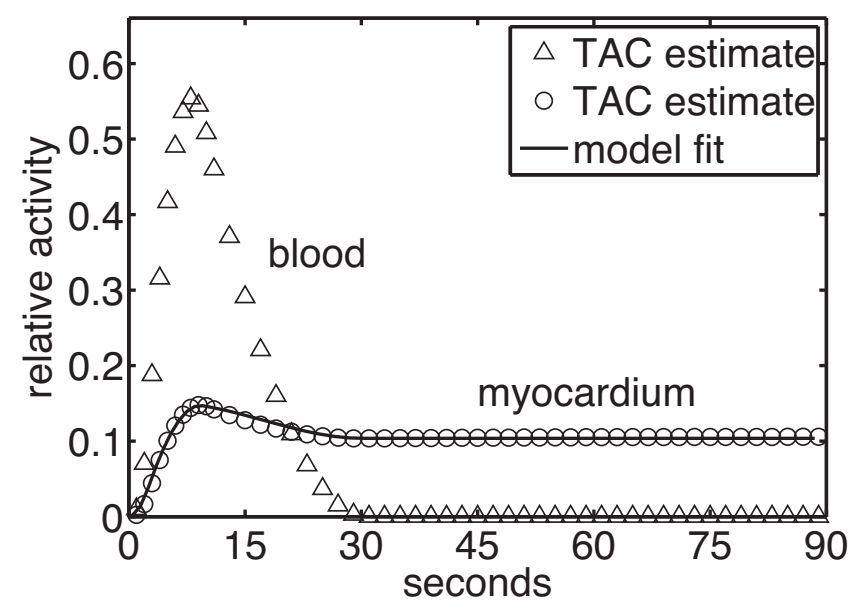

(b) result of tissue spillover correction

Fig. 5. TACs for an SHR estimated (a) without and (b) with tissue spillover correction for the blood curve. Spillover correction improves contrast between the blood input and myocardial uptake (triangles and circles, respectively), improves the fit of the compartmental model (solid line), and yields a metabolic rate estimate $\left(K_{i}\right)$ that nearly doubles, from $0.64 \mathrm{~min}^{-1}$ to $1.12 \mathrm{~min}^{-1}$.

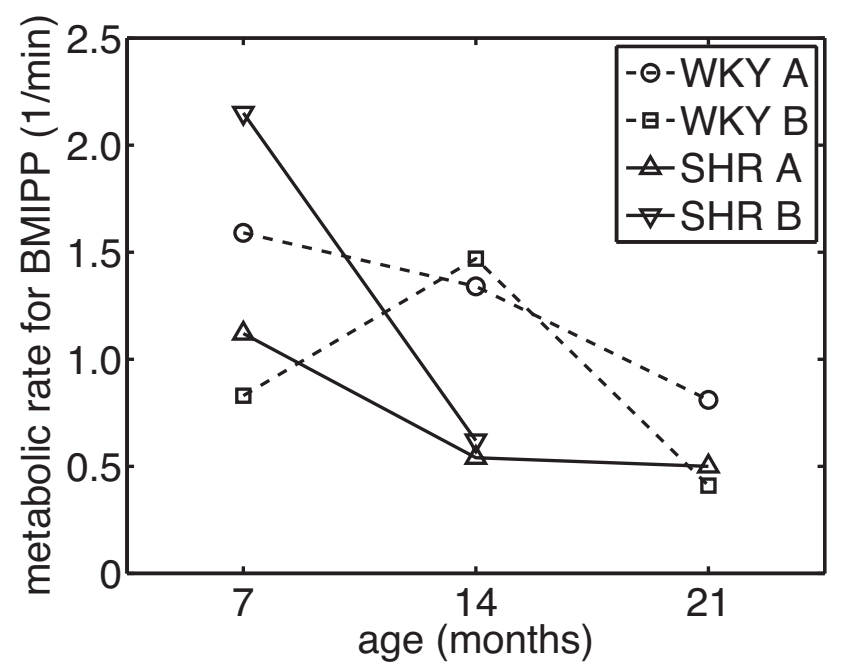

Fig. 6. Metabolic rate of ${ }^{123}$ I-BMIPP in the myocardium as a function of age. SHR B died of congestive heart failure before 21 months.

correction improved the contrast between the blood input and myocardial uptake curves for all studies and visually improved the fit of the compartmental model for some studies (Fig. 5). Metabolic rate estimates $\left(K_{i}\right)$ increased by an average of $72 \pm 45 \%$ across all 11 studies, compared to estimates obtained without spillover correction.

Estimates of $K_{i}$ obtained from corrected blood curves are plotted as a function of age for all 11 studies in Fig. 6. The general decline with age is what one expects. Slower rates of fatty acid metabolism in the SHRs at 14 months, compared to the WKY normal rats, is also expected as the SHR hearts switch to a reliance on glycolysis as the primary pathway for energy production during the development of heart failure. SHR B died of congestive heart failure before 21 months.

\section{DISCUSSION}

The tissue spillover correction method resulted in improved quantitative dynamic imaging of fatty acid metabolism in the rat heart, even with slow camera rotation.

Future work includes addressing computational issues associated with reconstructing a 4-D dynamic image from the entire $60 \mathrm{~min}$ of projection data. It is anticipated that an additional, reversible tissue compartment will be needed in the compartmental model to account for washout of ${ }^{123}$ I-BMIPP from the myocardium over this longer time scale.

\section{ACKNOWLEDGMENT}

This work was supported by the National Institutes of Health of the U. S. Department of Health and Human Services under grants R01-EB00121, R01-EB007219, and R01HL71253; and by the Director, Office of Science, Office of Biological and Environmental Research of the U. S. Department of Energy under contract DE-AC02-05CH11231.

This document was prepared as an account of work sponsored by the United States Government. While this document is believed to contain correct information, neither the United States Government nor any agency thereof, nor The Regents 
of the University of California, nor any of their employees, makes any warranty, express or implied, or assumes any legal responsibility for the accuracy, completeness, or usefulness of any information, apparatus, product, or process disclosed, or represents that its use would not infringe privately owned rights. Reference herein to any specific commercial product, process, or service by its trade name, trademark, manufacturer, or otherwise, does not necessarily constitute or imply its endorsement, recommendation, or favoring by the United States Government or any agency thereof, or The Regents of the University of California. The views and opinions of authors expressed herein do not necessarily state or reflect those of the United States Government or any agency thereof or The Regents of the University of California.

\section{REFERENCES}

[1] B. W. Reutter, G. T. Gullberg, R. Boutchko, K. Balakrishnan, E. H. Botvinick, and R. H. Huesman, "Fully 4-D dynamic cardiac SPECT image reconstruction using spatiotemporal B-spline voxelization," in 2007 IEEE Nuclear Science Symposium and Medical Imaging Conference Record, B. Yu, Ed., 2007, pp. 4217-4221.

[2] B. W. Reutter, R. Boutchko, R. H. Huesman, S. M. Hanrahan, K. M. Brennan, A. C. Sauve, and G. T. Gullberg, "Dynamic pinhole SPECT imaging and compartmental modeling of fatty acid metabolism in the rat heart," in 2008 IEEE Nuclear Science Symposium and Medical Imaging Conference Record, P. Sellin, Ed., 2008, pp. 4478-4481.

[3] B. W. Reutter, R. Boutchko, R. H. Huesman, A. C. Sauve, and G. T. Gullberg, "Penalized least-squares dynamic pinhole SPECT image reconstruction using a smooth 4-D image prior and multiresolution spatiotemporal B-splines," in Proceedings of the 10th International Conference on Fully Three-Dimensional Image Reconstruction in Radiology and Nuclear Medicine, B. M. W. Tsui and Z. Chen, Eds., 2009, pp. 110-113.

[4] R. H. Huesman, B. L. Knittel, B. M. Mazoyer, P. G. Coxson, E. M Salmeron, G. J. Klein, B. W. Reutter, and T. F. Budinger, "Notes on RFIT: A program for fitting compartmental models to region-of-interest dynamic emission tomography data," Lawrence Berkeley National Laboratory, Tech. Rep. LBL-37621, 1995.

[5] J. Hu, A. Sitek, B. W. Reutter, R. H. Huesman, and G. T. Gullberg, "A new approach of dynamic pinhole SPECT imaging for evaluation of sympathetic nervous system function in animal models of cardiac hypertrophy," in 2005 IEEE Nuclear Science Symposium and Medical Imaging Conference Record, B. Yu, Ed., 2005, pp. 2542-2546.

[6] A. Sauve, W. Choong, R. Boutchko, B. W. Reutter, A. Hwang, E. W. Izaguirre, B. H. Hasegawa, and G. T. Gullberg, "Quantitative imaging of cardiovascular function with pinhole SPECT in mice and rats - effects of attenuation and scatter," J Nucl Med, vol. 48, no. 5 suppl, pp. 424P-425P, 2007 (abstract).

[7] A. C. Sauve, B. W. Reutter, R. Boutchko, W.-S. Choong, R. H. Huesman, and G. T. Gullberg, "Multi-slice SPECT attenuation and scatter correction using Monte Carlo simulated system matrices," in 2008 IEEE Nuclear Science Symposium and Medical Imaging Conference Record, P. Sellin, Ed., 2008, pp. 4273-4276.

[8] B. W. Reutter, G. T. Gullberg, and R. H. Huesman, "Kinetic parameter estimation from attenuated SPECT projection measurements," IEEE Trans Nucl Sci, vol. 45, no. 6, pp. 3007-3013, 1998.

[9] B. W. Reutter, S. Oh, G. T. Gullberg, and R. H. Huesman, "Improved quantitation of dynamic SPECT via fully 4-D joint estimation of compartmental models and blood input function directly from projections," in 2005 IEEE Nuclear Science Symposium and Medical Imaging Conference Record, B. Yu, Ed., 2005, pp. 2337-2341. 